\title{
OCT4 promotes tumorigenesis and inhibits apoptosis of cervical cancer cells by miR-125b/BAK1 pathway
}

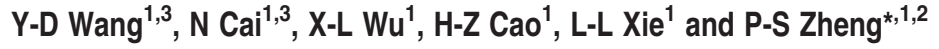

Octamer-binding transcription factor 4 (OCT4) is a key regulatory gene that maintains the pluripotency and self-renewal properties of embryonic stem cells. Although there is emerging evidence that it can function as oncogene in several cancers, the role in mediating cervical cancer remains unexplored. Here we found that OCT4 protein expression showed a pattern of gradual increase from normal cervix to cervical carcinoma in situ and then to invasive cervical cancer. Overexpression of OCT4 in two types of cervical cancer cells promotes the carcinogenesis, and inhibits cancer cell apoptosis. OCT4 induces upregulation of miR-125b through directly binding to the promoter of miR-125b-1 confirmed by chromatin immunoprecipitation analysis. MiRNA-125b overexpression suppressed apoptosis and expression of BAK1 protein. In contrast, miR-125b sponge impaired the anti-apoptotic effect of OCT4, along with the upregulated expression of BAK1. Significantly, Luciferase assay showed that the activity of the wild-type BAK1 $3^{\prime}$-untranslated region reporter was suppressed and this suppression was diminished when the miR-125b response element was mutated or deleted. In addition, we observed negative correlation between levels of BAK1 and OCT4, and positive between OCT4 and miR-125b in primary cervical cancers. These findings suggest an undescribed regulatory pathway in cervical cancer, by which OCT4 directly induces expression of miR-125b, which inhibits its direct target BAK1, leading to suppression of cervical cancer cell apoptosis.

Cell Death and Disease (2013) 4, e760; doi:10.1038/cddis.2013.272; published online 8 August 2013

Subject Category: Cancer

\section{Introduction}

Although Pap screen testing and early detection have dramatically increased survival rates, cervical cancer remains the second leading cause of cancer-related mortality among women worldwide. ${ }^{1}$ Epidemiological studies have suggested that multiple risk factors are involved in cervical carcinogenesis, with human papillomavirus regarded as the most dominant risk factor. ${ }^{2}$ However, the genetic and molecular events contributing to the initiation and progression of cervical cancer have been poorly elucidated. In recent years, studies have demonstrated that aberrant expression of certain stem cell-associated nuclear transcription factors, such as octamer-binding transcription factor 4 (OCT4), SOX2, NANOG and KLF4, could contribute to the tumorigenesis of various somatic cancers. ${ }^{3-5}$ However, the molecular mechanisms underlying these observations have not been fully explored.

OCT4 is a nuclear transcription factor of the POUhomeodomain family that has a critical role in several aspects of embryonic stem cell (ESC) maintenance, including ESC self-renewal, pluripotency and lineage commitment. ${ }^{6-8}$ Changes in OCT4 expression are involved in numerous developmental programs. For instance, a transient increase in OCT4 levels can induce lineage commitment to the primitive endoderm and mesoderm, whereas repression of OCT4 leads to trophectoderm differentiation. ${ }^{9,10}$ At the top of the pluripotent cell genetic regulatory network, OCT4 and SOX2 work cooperatively to stimulate the transcription of several target genes, including NANOG, FGF-4, UTF1, Fbx15, microRNA-302 clusters and even Sox2 and Oct4 themselves. ${ }^{11-15}$ Consistent with their roles in maintaining pluripotency, overexpression of specific transcription factors (Oct4, Sox2, Klf4 and c-Myc) can induce somatic cells to acquire pluripotency. These induced pluripotent stem cells have characteristics similar to ESCs. ${ }^{16}$ It was recently proposed that OCT4 acts as a multi-functional factor during cancer development. Hochedlinger et al. reported that ectopic OCT4 expression in somatic cells causes epithelial dysplasia. ${ }^{17}$ In addition, OCT4 has been detected in germ cell tumors ${ }^{18-20}$ and various human somatic tumors, including hepatoma, ${ }^{21}$ breast cancer ${ }^{22,23}$ and bladder cancer, ${ }^{24}$ suggesting that OCT4 functions in both the embryo and the adult. However, no study has yet defined a potential function for OCT4 in cervical cancer.

In the present study, we found that OCT4 was upregulated in cervical lesions and that exogenous expression of OCT4 in

\footnotetext{
${ }^{1}$ Department of Reproductive Medicine, First Affiliated Hospital, Xi'an Jiaotong University of Medical School, Xi'an, People's Republic of China and ${ }^{2}$ Section of Cancer Stem Cell Research, Key Laboratory of Environment and Genes Related to Diseases, Ministry of Education of People's Republic of China, Xi'an, People's Republic of China

*Corresponding author: P-S Zheng, Department of Reproductive Medicine, First Affiliated Hospital, Xi'an Jiaotong University of Medical School, Xi'an 710061, People's Republic of China. Tel: + 86298265 7874; Fax: + 86298532 4013; E-mail: zpsheng @mail.xjtu.edu.cn

${ }^{3}$ These authors contributed equally to this work.

Keywords: OCT4; cervical cancer; apoptosis; miR-125b-1; BAK1

Abbreviations: ChIP, chromatin immunoprecipitation; CIS, cervical carcinoma in situ; ESCs, embryonic stem cells; ICC, invasive cervical carcinoma; IHC, immunohistochemistry; IRS, immunoreactivity scores; miRNAs, microRNAs; MRE, miR-125b response element; NC, normal cervix; OCT4, octamer-binding transcription factor 4; PBS, phosphate-buffered saline; RRs, regulatory regions; RT-PCR, reverse transcription-PCR; UTR, untranslated region

Received 13.11.12; revised 15.6.13; accepted 19.6.13; Edited by H-U Simon
} 
cervical cancer cells enhanced tumor formation. The ability of OCT4 to potentiate tumor growth was mediated, at least in part, by an inhibition of apoptosis mediated by OCT4-induced transactivation of miR-125b, which, in turn, directly targets BAK1. These findings support the hypothesis that OCT4 works as an oncogene in cervical carcinogenesis.

\section{Results}

OCT4 expression in human normal cervical (NC) epithelium and cervical lesions. OCT4 expression has been detected in various human germ cell tumors and somatic carcinomas, including hepatocellular carcinoma, breast carcinoma and bladder cancer. However, the potential relationship between OCT4 protein levels and cervical carcinoma has not yet been explored. In the present study, immunohistochemistry (IHC) was used to investigate OCT4 expression in different human cervical epithelial lesions (Figure 1a). OCT4-positive cells were found in $35.71 \%$ (15/42) of NC samples, $75.00 \%$ (15/20) of cervical carcinoma in situ (CIS) samples and $88.64 \%$ (39/44) of invasive cervical carcinoma samples (Figure 1b). The average immunoreactivity scores (IRSs) for OCT4 staining were $4.74 \pm 0.67$ in $\mathrm{NC}(n=42), 7.25 \pm 0.76$ in CIS $(n=20)$ and $10.64 \pm 0.63$ in invasive cervical cancer (ICC; $n=44$; Figure 1c, NC versus
CIS, $P=0.027$; CIS versus ICC, $P=0.0030$; NC versus ICC, $P=1.61 \mathrm{E}-09$; one-way ANOVA test), implying that OCT4 may be upregulated during cervical carcinogenesis. To further confirm this expression pattern, western blot analyses were performed using samples of normal $(n=8)$ and cervical carcinoma tissues $(n=8$; Figure $1 \mathrm{~d})$. The average OCT4 expression level was significantly lower in normal samples $(0.30 \pm 0.084)$ relative to cervical carcinoma tissue (0.75 \pm 0.11 ; Figure 1e, $P=0.0055$, Student's $t$-test). Furthermore, the OCT4 expression in original lesions of cervical cancer patients with or without lymphatic metastasis was analyzed. As shown in Table 1, statistically, OCT4 expression was significantly higher in primary lesions with lymphatic metastasis than those without lymphatic metastasis, indicating that OCT4-positive cells may have an important role in metastasis. Consistent with our results, recent studies have demonstrated that OCT4 expression was associated with cell motility and migration. ${ }^{25,26}$

It is well known that high-risk HPVs (such as HPV16 and HPV18) infection is considered to be a risk factor for cervical cancer. Then the viral DNA was detected in cervical cancer patients using universal primers GP5 + $6+$, which can detect 22 types of HPV. ${ }^{27} 81.82 \%$ (36/44) of the patients are infected with HPV. And the OCT4 IRS is, respectively, $9.00 \pm 1.55$ in $\mathrm{HPV}^{-}$and $11.00 \pm 0.69$ in $\mathrm{HPV}^{+}$samples, indicating that
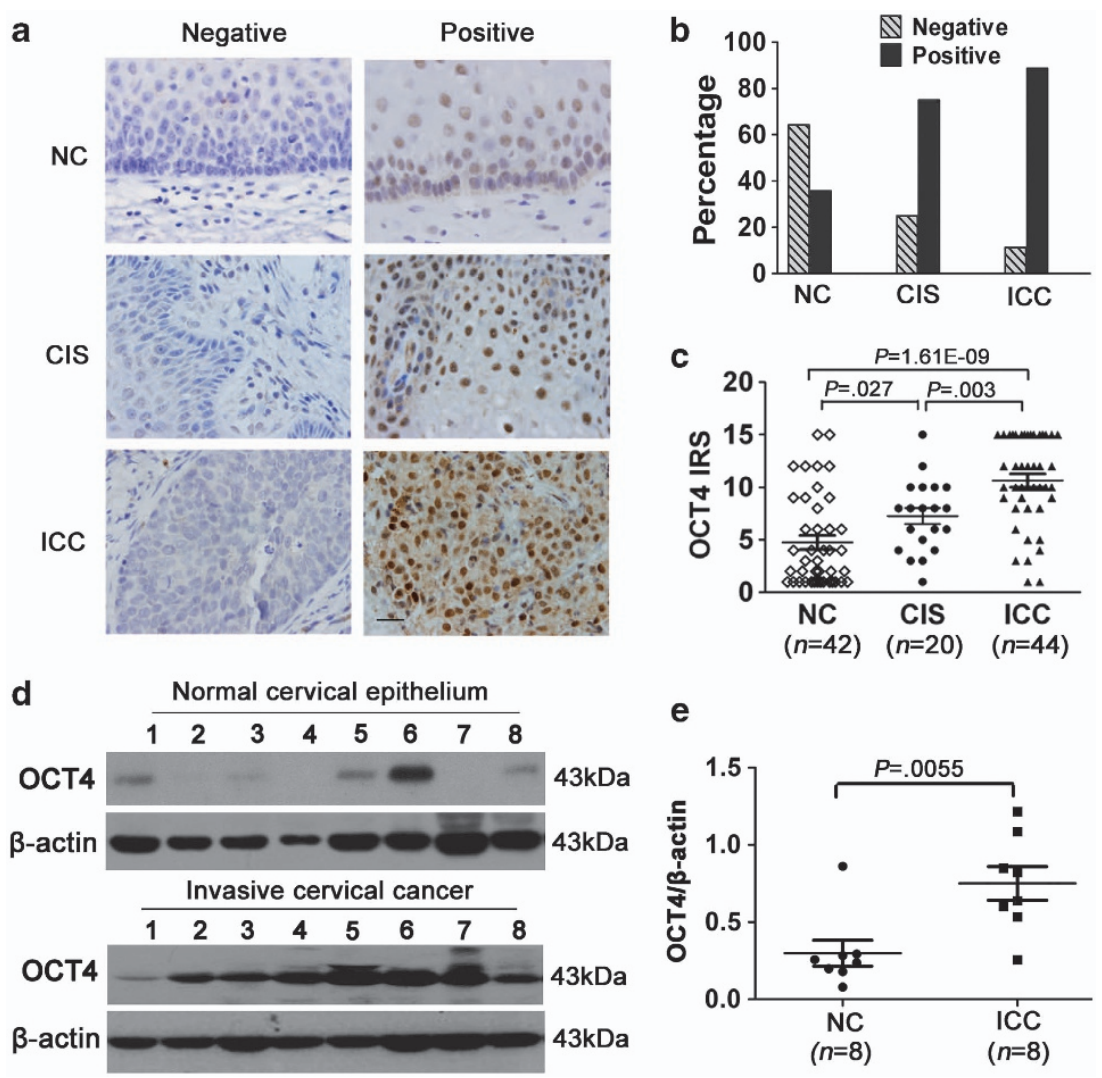

Figure 1 OCT4 protein expression in normal cervical (NC) tissue and cervical cancer lesions. (a) Representative immunostained specimens showing OCT4 expression in NC $(n=42)$, cervical carcinoma in situ (CIS; $n=20$ ) and invasive cervical carcinoma (ICC; $n=44)$. Scale bars $=20 \mu \mathrm{m}$. (b) Bar chart showing the frequency of OCT4-positive samples among different cervical lesions. (c) The scatter plot shows the immunoreactivity scores (IRS) for OCT4 staining in tissue samples (NC versus CIS, $P=0.027$; CIS versus ICC, $P=0.0030$; NC versus ICC, $P=1.61 \mathrm{E}-09$, one-way ANOVA test). (d) Western blot analysis of OCT4 protein levels in NC ( $n=8)$ and ICC ( $n=8)$ specimens. (e) Ratio of OCT4 to $\beta$-actin staining density in NC and ICC specimens determined with the AlphaView SA software $(P=0.0055$; Student's $t$-test) 
Table 1 OCT4 expression in cervical cancer patients with different status

\begin{tabular}{|c|c|c|c|c|}
\hline Groups & $n$ & $\begin{array}{c}\text { OCT4 IRS } \\
(\bar{X} \pm \mathrm{S})\end{array}$ & $t$ & $P^{a}$ \\
\hline Primary site without HPV & 8 & $9.00 \pm 1.55$ & & \\
\hline Primary site with HPV & 36 & $11.00 \pm 0.69$ & 1.22 & 0.23 \\
\hline $\begin{array}{l}\text { Primary site without lymphatic } \\
\text { metastasis }\end{array}$ & 32 & $9.59 \pm 0.77$ & & \\
\hline Primary site with lymphatic & 12 & $13.42 \pm 0.60$ & 2.91 & 0.0058 \\
\hline
\end{tabular}

astudent's $t$-test.

there is no significant difference of OCT4 expression between the HPV-positive and -negative samples (Table 1).

OCT4 promotes tumor formation by cervical cancer cells in vivo. Endogenous OCT4 protein levels were examined by western blotting and IHC in three cervical cancer cell lines (Figures 2a and b). OCT4 protein was low in C-33 A cells and undetectable in HeLa and $\mathrm{SiHa}$ cells. To investigate the function of OCT4 in cervical cancer cells, a human OCT4-expressing plasmid was constructed (Supplementary Figure 3a1) and overexpressed in HeLa and SiHa cells by stable transfection (Figure 2c). Chang et al. ${ }^{28}$ found that OCT4 could regulate tumorigenic properties in human colorectal cancer cells. Tumor formation assays performed in athymic nude mice were carried out to explore the role of OCT4 in mediating cervical carcinogenesis and indicated that tumors formed by OCT4-expressing cells were greater in volume (Figures 2d and e, $P<0.05$, two-way ANOVA test) and weight (Figures $2 f$ and $g, P=0.0048$ and 0.0055 , respectively, Student's $t$-test) than tumors formed by control cells, suggesting that OCT4 promotes tumor formation in cervical cancer cells.

Then the expression of OCT4 and $\mathrm{Ki}-67$ protein was examined by $\mathrm{IHC}$ in xenograft tumor tissues (Figure 2h). OCT4 was expressed in tumor tissues formed by HeLa-OCT4 cells, but the levels of Ki-67 had no aberrant change. And similar results were observed in $\mathrm{SiHa}$ cells, indicating that OCT4 had no relationship to cell proliferation in vivo and the proliferative potential did not contribute to the promotion of tumor formation.

OCT4 inhibits cervical cancer cell apoptosis in vitro and in vivo. OCT4 has been reported to affect apoptosis in murine ESCs and human cancer stem cell-like cells. ${ }^{29,30}$ The frequency of apoptosis of cervical cancer cells in vitro was measured with a flow cytometry-based apoptosis assay. As shown in Figure 3a, a significant decrease in the proportion of apoptotic cells was observed among HeLa-OCT4/SiHa-OCT4 cells relative to the corresponding control cells $(P<0.05$, Student's $t$-test), suggesting that OCT4 inhibited spontaneous apoptosis in these cervical cancer cell lines in vitro.

Furthermore, TUNEL assays were performed on tissue sections from the xenografted tumors. Tumors formed by OCT4-expressing cells contained fewer apoptotic cells than control cells (Figure $3 \mathrm{~b}, P<0.05$, Student's $t$-test), indicating that OCT4 may promote tumor formation by inhibiting apoptosis.
OCT4 inhibits apoptosis by transactivating miR-125b through its interaction with miR-125b-1 promoter. To further explore the mechanism by which OCT4 inhibits apoptosis in cervical cancer cells, we determined the expression levels of several apoptosis-related genes (Supplementary Figure 1) and microRNAs (miRNAs; Figure 4a) in each cell line. Interestingly, miR-125b was the only unique gene that was consistently upregulated by at least twofold in HeLa-OCT4 and SiHa-OCT4 cells relative to controls, indicating that OCT4 might transactivate miR-125b.

MiRNA-125b is a primary oncogenic factor that is transcribed from two loci located on chromosomes 11q24 (hsa-miR-125b-1) ${ }^{31}$ and 21q21 (hsa-miR-125b-2). ${ }^{32}$ We therefore analyzed whether miR-125b-1 and/or -2 were activated by OCT4 using real-time PCR. The level of pri-miR-125b-1 was consistently increased upon OCT4 overexpression in two cell lines (Figure 4b, $P=0.042$ and 0.020 , respectively, Student's $t$-test). In contrast, pri-miR-125b-2 was only increased in HeLa-OCT4 cells (Figure 4c, $P=0.042$, Student's $t$-test), but not significantly affected in SiHa-OCT4 cells ( $P=0.78$, Student's $t$-test). Therefore, miR-125b-1 induced by OCT4 may contribute to the upregulation of mature miR-125b.

Luciferase reporter assays were performed to confirm whether miR-125b was transactivated by OCT4 via the miR$125 \mathrm{~b}-1$ promoter in cervical cancer cells. We cloned 5-kb DNA sequences flanking the miR-125b-1 promoter region. Sequence analyses revealed the presence of two conserved octamer regulatory regions (RRs) in this region, named RR1 (between $-4280 \mathrm{nt}$ and $-4287 \mathrm{nt}$, pink oval) and RR2 (between $-439 \mathrm{nt}$ and $-446 \mathrm{nt}$, red oval; Figure $4 \mathrm{~d}$ ). We established a series of promoter deletions, including fragments lacking -5000 to -2700 (RR1), -5000 to -700 (RR1), -5000 to -300 (RR1 and RR2) and rearrangements containing only RR1 or both RR1 and RR2. The luciferase assays (Figure $4 \mathrm{~d}$ and Supplementary Figure 2 ) revealed that the full-length miR-125b-1 promoter and the promoters lacking nucleotides -5000 to -700 showed statistically significant activity in response to OCT4 expression in both HeLa and $\mathrm{SiHa}$ cells. However, luciferase activity was completely absent when the fragment lacking the sequence upstream of position -300 was tested $(P=0.71$ and 0.59 , respectively, Student's $t$-test). And RR2 induced significant luciferase activity in both HeLa and SiHa cells $(P=0.0018$ and 0.041 , respectively, Student's $t$-test), whereas RR1 was only active in HeLa cells ( $P=0.0036$, Student's $t$-test), but not in SiHa cells $(P=0.91$, Student's $t$-test). These data suggest that the region RR2 contains a key transcriptional element.

Chromatin immunoprecipitation (ChIP) assays were conducted to determine whether OCT4 controls miR-125b-1 expression by directly binding to response elements within the miR-125b-1 promoter. OCT4 bound to both the RR1 and RR2 regions in HeLa cells (Figure 4e, $P=0.036$ and 0.035 , respectively, Student's $t$-test), but only to RR2 in SiHa cells ( $P=0.0030$, Student's $t$-test). Thus, OCT4 specifically binds to its putative response elements in the miR-125b-1 promoter, providing strong evidence that miR-125b-1 can be directly regulated by OCT4.

To further confirm that miR-125b mediates the OCT4-induced inhibition of apoptosis in cervical cancer cells, 

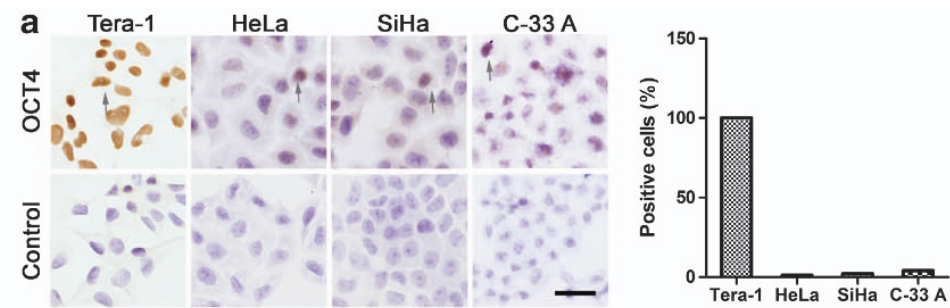

b

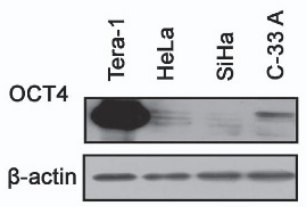

d

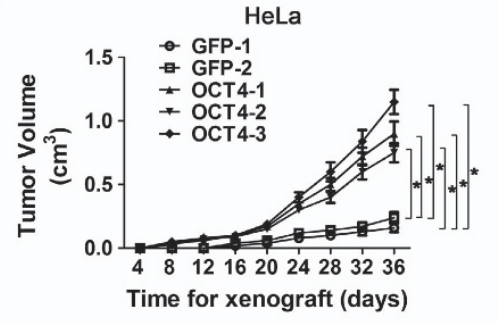

f

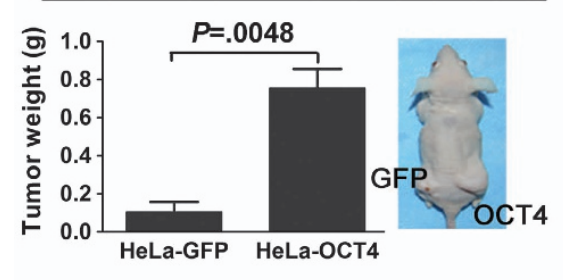

c
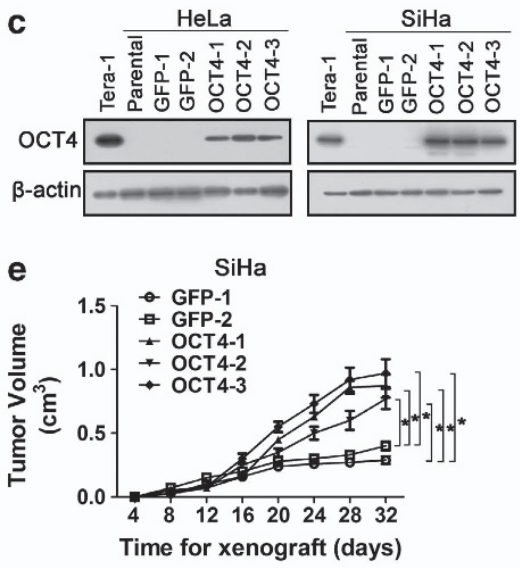

g

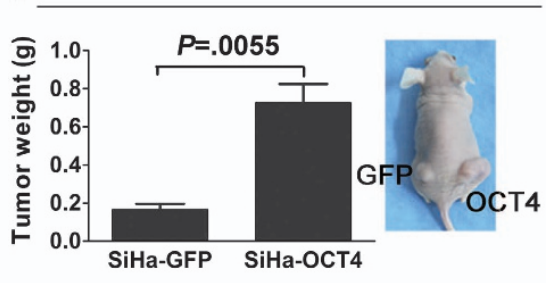

h

hela-GFP HeLa-OCT4

SiHa-GFP SiHa-OCT4

ঠু

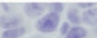

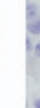

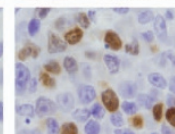

\&ing $e^{2}$

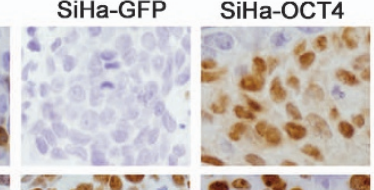

$\sqrt{0}$

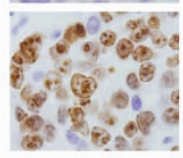

音
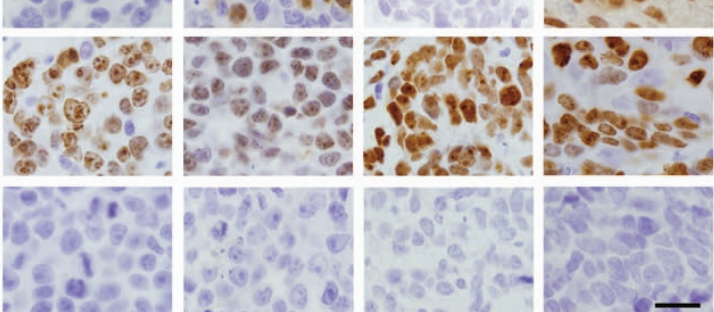

.

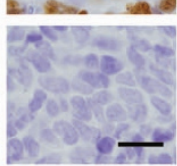

Figure 2 OCT4 promotes tumor formation by cervical cancer cells in vivo. (a) OCT4 expression in cervical cancer cell lines was detected by immunocytochemistry and the positive rates were summarized. The arrow indicates the OCT4 positive cell. (b) OCT4 expression in cervical cancer cell lines was detected by western blotting. (c) Western blot analysis of OCT4 protein and in HeLa and SiHa parental, control- and OCT4-stably transfected cells. For western blot analyses, Tera-1 cells were used as a positive control, and $\beta$-actin served as the loading control. A tumor formation assay was performed with three mice per group. Tumor growth curves were calculated based on monitoring performed every 4 days post-transplant. At 36 days post-transplant for HeLa cells (d) and 32 days post-transplant for SiHa cells (e), the xenograft tumors were dissociated and weighed ( $\mathbf{f}$ and $\mathbf{g}$ ). The data were analyzed and shown as mean \pm S.E.M. (d and $\mathbf{e}$ were determined by two-way ANOVA test, ${ }^{*} P<0.05$ whereas $\mathbf{f}$ and $\mathbf{g}$ were determined by Student's $t$-test). (h) Serial sections of immunochemical staining for OCT4 and Ki-67 in indicated tumor xenografts. Scale bar $=20 \mu \mathrm{m}$

miR-125b overexpression and sponge vectors were constructed and identified by real-time PCR or dual luciferase assay (Supplementary Figure 3). Then miR-125b overexpression or sponge plasmid was transiently transfected into OCT4 overexpressing and control cells. Flow cytometry-based apoptosis assay shows that apoptotic cells were 2-fold decrease or 1.5-fold increase in HeLa-GFP cells transfected with the
miR-125b expression or sponge plasmid, compared with HeLa-GFP cells transfected with the control (Figure 4f). In addition, miR-125b sponge restored the depression of apoptosis by OCT4 overexpression. Taken together, these results indicate that HeLa cell apoptosis was negatively correlated with miR-125b expression, and inactivation of miR-125b can abolish the effect induced by OCT4. Similar results were found in SiHa cells, 

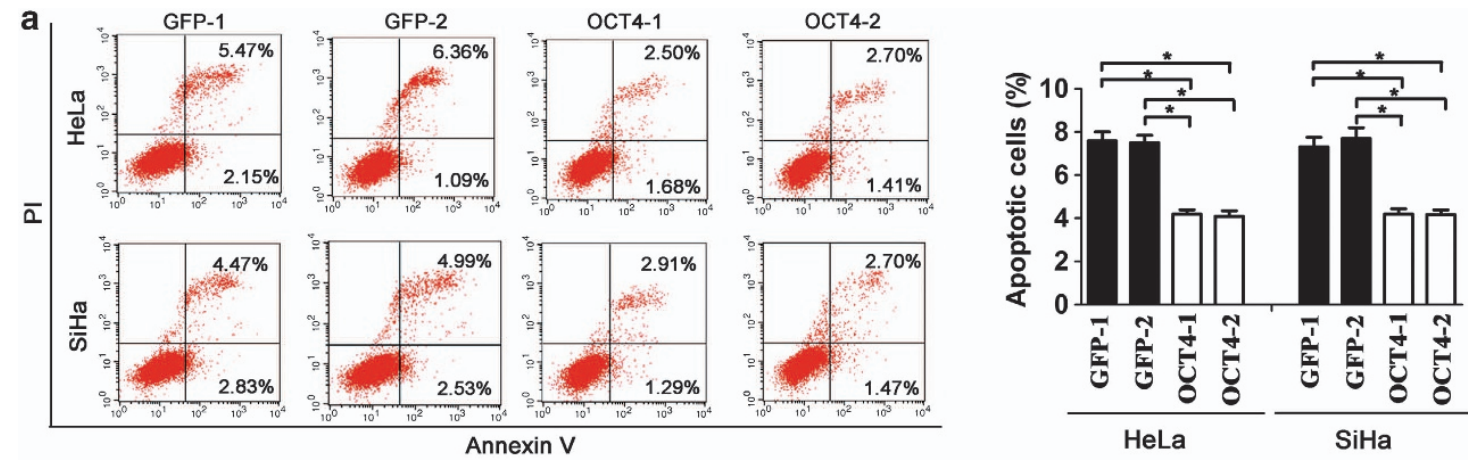

b
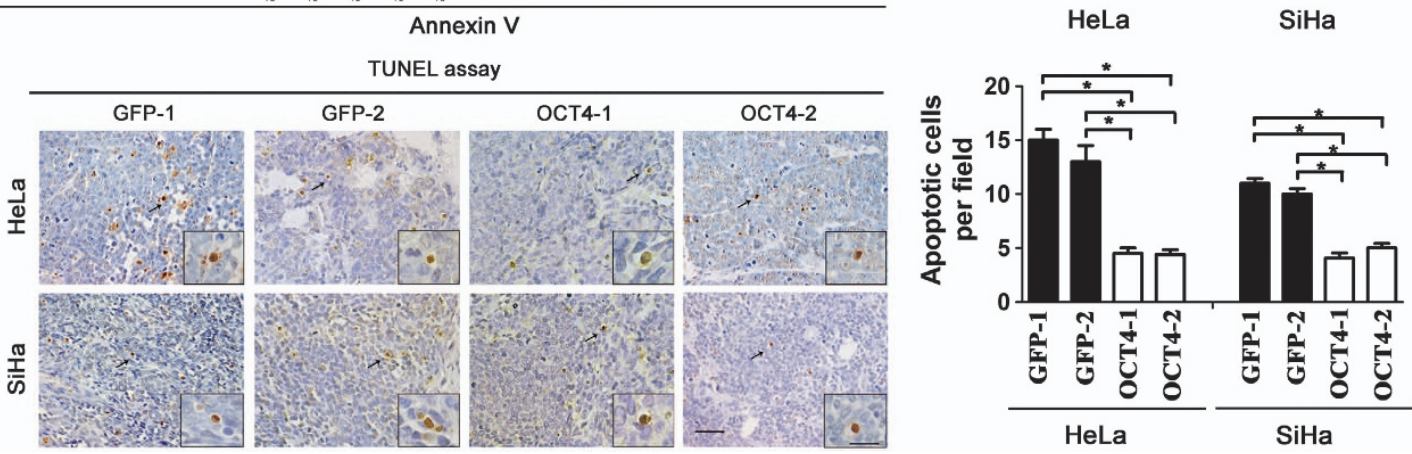

Figure 3 Exogenous OCT4 expression inhibits apoptosis of human cervical cancer cells in vitro and in vivo. (a) Apoptosis analysis based on flow cytometry was performed triplicate. All of the data were summarized and presented as the mean \pm S.E.M. (Student's $t$-test), ${ }^{*} P<0.05$. (b) TUNEL assays were performed triplicate using the tumors from Figures $2 \mathrm{~d}$ and e. Scale bar $=50 \mu \mathrm{m}$ for main image, $20 \mu \mathrm{m}$ for inset; arrows indicate representative TUNEL + cells. And the data were analyzed and presented as the mean \pm S.E.M. (Student's $t$-test), ${ }^{*} P<0.05$

suggesting that OCT4 inhibits apoptosis via the upregulation of miR-125b in cervical cancer cells.

Exogenous OCT4 downregulates BAK1 by transactivating miR-125b in cervical cancer cells. Recently, Zhou et al. reported that BAK1 was a direct target of miR$125 \mathrm{~b}$ in breast cancer cells. ${ }^{33}$ BAK1 protein was detected by western blot analysis. Although BAK1 mRNA has no change in both HeLa-OCT4 and SiHa-OCT4 cells (Supplementary Figure $3, P=0.84$ and 0.81 , respectively, Student's $t$-test), BAK1 protein was downregulated in OCT4-expressing cells (Figure $5 \mathrm{a}$ ), indicating that decline of BAK1 protein was not directly mediated at transcriptional level by OCT4. Indeed, we found miR-125b response element (MRE) in BAK1 mRNA was highly conserved among human, mouse and rat (Figure 5b). To further clarify the relationship between OCT4 and BAK1 in cervical cancer, we compared BAK1 protein levels in miR-125b-overexpressing HeLa-GFP and SiHa-GFP cells, and miR-125b-sponge-transfected HeLaOCT4 and SiHa-OCT4 cells (Figure 5c). MiR-125b overexpression led to downregulation of BAK1. In contrast, miR-125b sponge induced more than twofold increases in BAK1 levels within OCT4-expressing cells. Therefore, OCT4 overexpression in the cervical cancer cell lines downregulated BAK1 by transactivaton of miR-125b. Furthermore, to confirm the function of miR-125b in the mediation of BAK1 by OCT4, the $3^{\prime}$-untranslated region (UTR) of wild type of $B A K 1$ (BAK1wt) was inserted downstream of a luciferase vector. Remarkably, the luciferase activity was repressed in HeLa-OCT4 cells compared with that in control cells, with a repression rate of more than $40 \%$. The constructs containing the mutated or deleted sequence of miR-125b-binding site
(BAK1mut or BAK1del) were produced as a control. The Luciferase activity measurements indicated specific repression of the wild-type substrate by OCT4 and no effect when the MRE was mutated or deleted (Figure $5 d$ ), suggesting that BAK1 was the direct target of miR-125b and that miR-125b is critical for the OCT4-mediated regulation of BAK1 expression. In addition, in 20 human cervical cancer samples (Figure 5e), levels of cytoplasmic BAK1 were inversely correlated with nuclear OCT4 expression (Figure 5f), indicating that BAK1 downregulation also occurred in OCT4reactivated human cervical carcinoma tissues. Furthermore, miR-125b was detected in 13 clinical samples and it was positively correlated with OCT4 (Figure $5 \mathrm{~g}$ ). All together, these results suggest that OCT4 promotes cervical cancer development by inhibiting apoptosis via miR-125b repression of BAK1 (Figure 6).

Knocking down OCT4 in PA-1 cells resulted in apoptosis and inhibited miR-125b expression. To further explore the role of OCT4 in apoptosis, the OCT4 was knocked down in $\mathrm{PA}-1$, which is a human ovarian teratoma cell line with high OCT4 expression. As shown in Supplementary Figure 4, after OCT4 inhibition, the level of miR-125b was downregulated, BAK1 was upregulated and the proportion of apoptotic cells were significantly increased, emphasizing the notion that OCT4 regulates cell apoptosis by activating miR-125b.

\section{Discussion}

OCT4 appears to be active in many human somatic tumors, but its possible role in cervical carcinoma has not been 


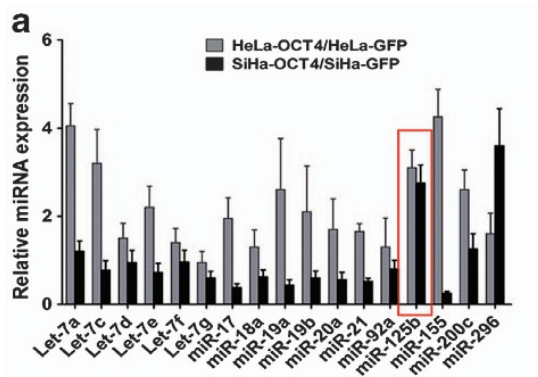

b

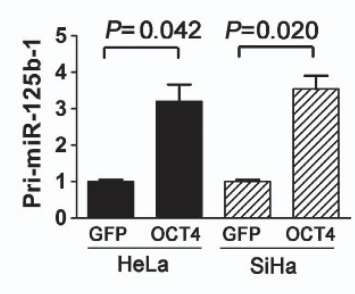

c

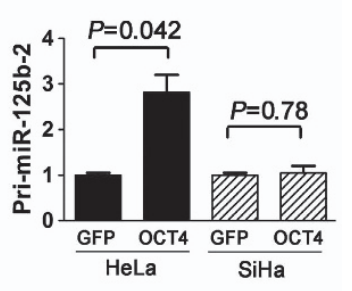

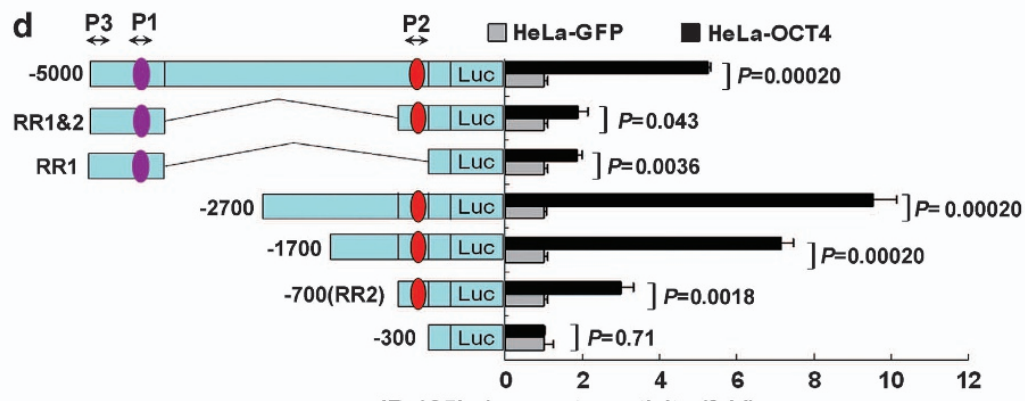

miR-125b-1 promoter activity (fold)
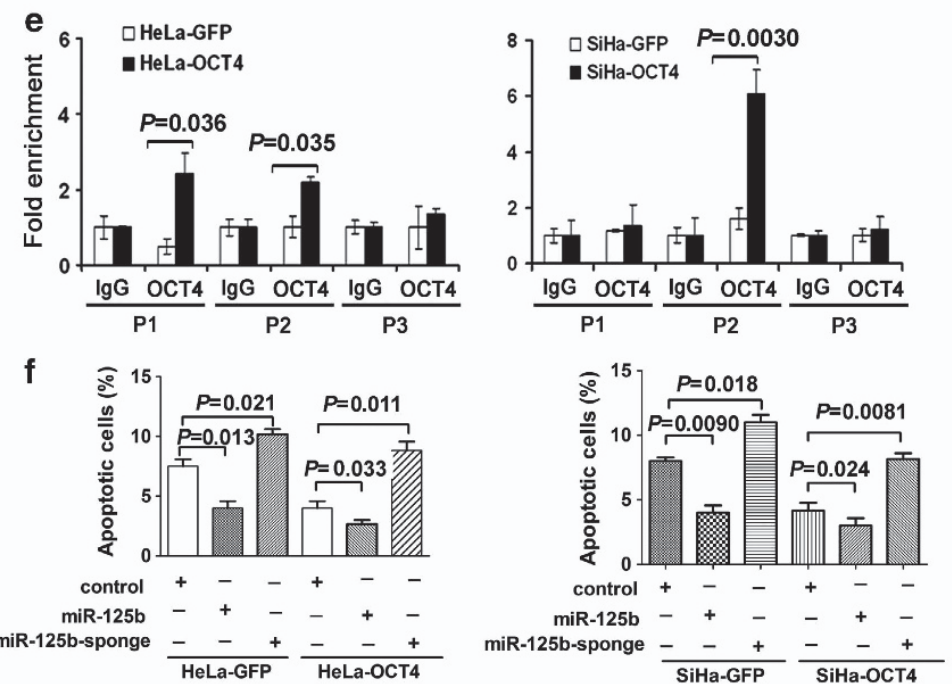

Figure 4 Exogenous OCT4 expression inhibits apoptosis by transactivating miR-125b-1. (a) Apoptosis-related miRNAs were screened by real-time PCR in control and OCT4-expressing HeLa and SiHa cells. Real-time PCR analysis of pri-miR-125b-1 (b) and pri-miR-125b-2 (c) in OCT4-expressing and control cells. Data are presented as the mean \pm S.E.M. of triplicate analyses (Student's t-test). (d) Schematic structure of miR-125b-1 promoter. The pink and red ovals indicate the location of two potential OCT4binding sites (RR1 and RR2). The bidirectional arrows (P1, P2 and P3) represent the primers used in ChIP analysis. Dual luciferase reporter assays in HeLa-GFP/OCT4 cells with a series of miR-125b-1 promoter $5^{\prime}$ deletion and rearrangement mutants. Data are presented as the mean \pm S.E.M. of triplicate analyses (Student's $t$-test). (e) Quantitative ChIP analysis of OCT4 binding to miR-125b-1 promoter sequences is shown for HeLa-GFP/OCT4 and SiHa-GFP/OCT4 cells. Data are presented as the mean \pm S.E.M. of triplicate analyses (Student's $t$-test). (f) Apoptosis assays were performed on OCT4-expressing or control cells following transfection with the miR$125 \mathrm{~b}$-expressing or -sponge vector. Data are presented as the mean \pm S.E.M. of triplicate analyses (Student's $t$-test)

investigated to date. In the present study, we show that OCT4 is upregulated in cervical carcinomas and invasive cervical carcinomas relative to $\mathrm{NC}$ tissue by both $\mathrm{IHC}$ and western blot analyses (Figure 1). OCT4 was similarly found to be upregulated in hepatocellular carcinoma ${ }^{34}$ and gastric cancer. ${ }^{35}$ These results suggest that OCT4 reactivation in cancer cells might contribute to the development and progression of these types of cancer. Furthermore, OCT4 is recognized as an ESC-specific protein ${ }^{8,36}$ and has frequently been described as a marker of germ cell tumors, including teratomas, ${ }^{19,20,37}$ thereby suggesting that OCT4-positive cells may be cancer stem cells in germ cell carcinomas. However, it is necessary to gather more data in support of the notion that OCT4-positive cells are the tumor-initiating cells in somatic carcinomas. Nevertheless, OCT4 protein cannot be detected in most somatic cancer cell lines, which may reflect the inappropriate use of teratoma cells as a positive control. ${ }^{38}$ We found that OCT4 expression in somatic cancer cells is clearly weaker than that in germ line carcinoma cells (Figure 2a).

To further explore the effect of OCT4 on cervical tumorigenesis, exogenous OCT4 was stably transfected into two 


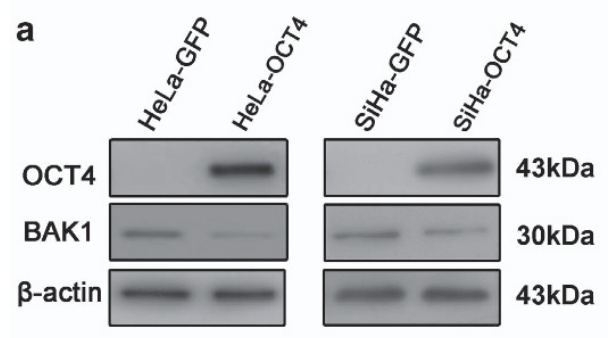

b

C

Rattus norvegicus ...CAGTCCTCTCCTGCCUCUCAGGGC... Mus musculus ...CUUGGCGGCUGGACUCUCAGGGA... Homo sapiens 5'...CUUUGCAGUUGGACUCUCAGGGA...

miR-125b

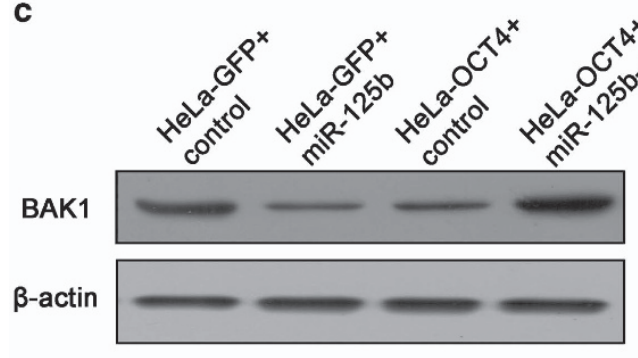

d
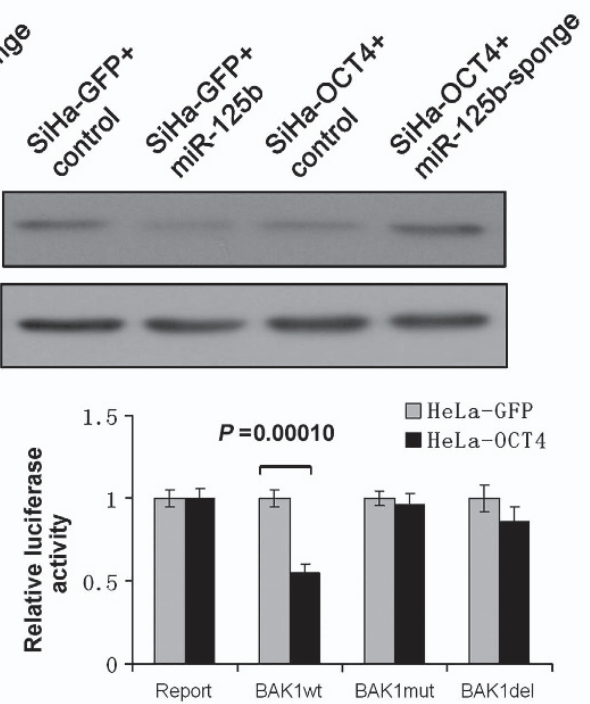

e
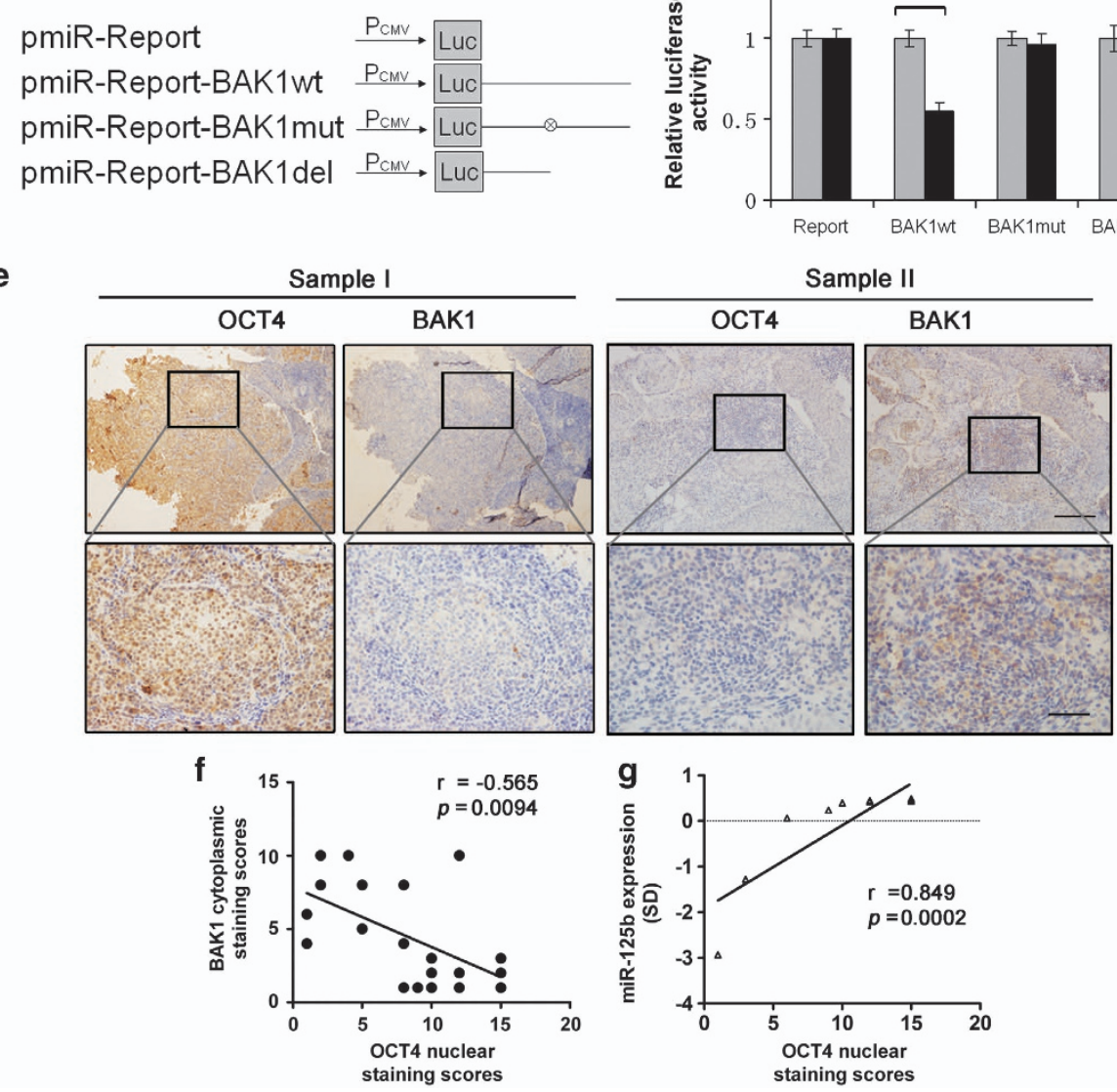

Figure 5 Exogenous OCT4 expression downregulates BAK1 through the activation of miR-125b. (a) BAK1 expression was detected in the indicated cell lines by western blot analysis. (b) Highly conserved, predicted binding sites for the seed sequences of miR-125b among human (629-636), mouse (607-614) and rat (87-94) in the 3'-UTR (untranslated region) of BAK1. (c) BAK1 protein levels were determined by western blot analysis in HeLa-GFP or SiHa-GFP cells following transfection with the miR-125b1-expressing or control vector and in HeLa-OCT4 or SiHa-OCT4 cells transfected with the miR-125b-sponge or control vector. (d) Schematic representation of the constructs used in the luciferase assay. HeLa-GFP/OCT4 cells were transfected with plasmid of BAK1wt, BAK1mut or BAK1del. Dual luciferase reporter activity was expressed relative to the pmiR-Report vector and data are presented as the mean \pm S.E.M. of triplicate analyses (Student's $t$-test). (e) Expression of OCT4 and BAK1 was examined by IHC staining in 20 cervical cancers specimens. The expression levels of two representative samples are shown. Scale bars $=200 \mu \mathrm{m}$ for upper panel, $50 \mu \mathrm{m}$ for lower panel. (f) Nuclear OCT4 and cytoplasmic BAK1 staining were scored from 1 to 15. The correlation was significant, as determined by the Pearson's correlation test $(r=-0.565$; $P=0.0094)$. (g) Expression of miR-125b was detected in 13 clinical samples from Figure 1 by real-time PCR and the correlation between OCT4 and miR-125b was significant, as determined by the Pearson's correlation test $(r=0.849 ; P=0.0002)$ 


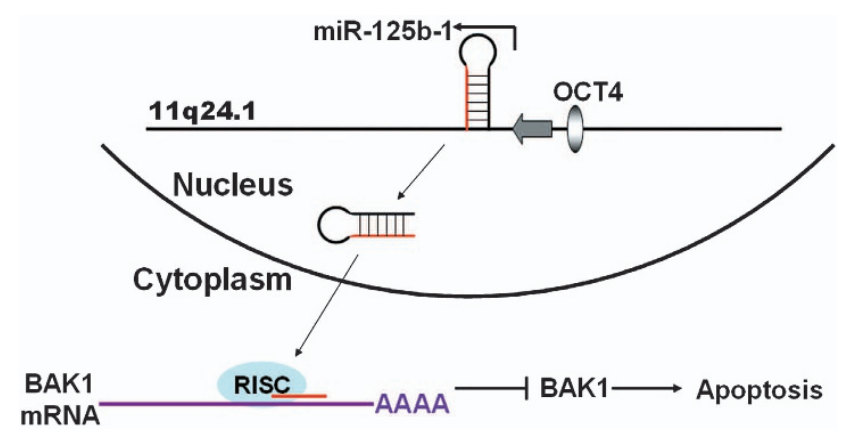

Figure 6 A model of the OCT4/miR-125b/BAK1 pathway in human cervical carcinogenesis. OCT4 expression activates miR-125b-1. In turn, miR-125b-1 inhibits BAK1 translation, which inhibits apoptosis and promotes tumor progression

cervical cancer cell lines. Exogenous OCT4 expression substantially enhanced the tumor formation properties of these cervical carcinoma cells in vivo (Figure 2). In accord with this result, Chang et al. ${ }^{28}$ found that OCT4 overexpression could promote tumor formation by human colorectal cancer cells, and $\mathrm{Kim}$ and $\mathrm{Nam}^{39}$ determined that Oct4 in a mouse model of breast cancer enhanced tumorigenesis and increased the number of cancer stem cells. Combined with our findings, these studies suggest that OCT4 can promote the progression of cancer.

How does OCT4 enhance tumor formation in models of cervical carcinoma? Exogenous expression of OCT4 in cervical cancer cells did not enhance cell proliferation potential in vivo (Figure 2h). However, apoptosis was inhibited in OCT4-expressing cells and xenograft tumor tissues formed by OCT4-expressing cells, and increased in OCT4-knocked down PA-1 cells, as visualized by FACS analysis and TUNEL assays, respectively (Figure 3 and Supplementary Figure 4b). In murine lung carcinoma and human breast cancer cell lines, knockdown of Oct4 expression by small interfering RNA results in apoptosis of cancer stem cell-like cells through the Oct4/Tcl1/Akt1 pathway. ${ }^{29}$ In mouse ESCs, knockdown of Oct4 expression induced apoptosis via the Stat3/survival pathway ${ }^{30}$ or the Trp53 pathway..$^{40}$ The present findings are consistent with these reports, supporting the notion that OCT4 functions as an anti-apoptotic gene in human and murine stem cells and cancer cells. Nonetheless, the pathway controlling the apoptotic process may depend on the activation state of the cell.

In the present study, OCT4 bound to and transactivated the miRNA-125b-1 promoter, upregulating miRNA-125b expression in cervical cancer and teratocarcinoma cells (Figures $4 a-e$ and Supplementary Figure $4 b$ ). The OCT4mediating increase in miRNA-125b levels led to inhibition of apoptosis (Figure 4f). This is the first report to show that OCT4 can inhibit apoptosis by transactivating miRNA-125b. As one of the earliest miRNAs to be discovered, miRNA-125b is a primary oncogenic factor that is involved in proliferation, apoptosis, differentiation, drug resistance and immunity. Recently, miRNA-125b has been studied in many tumors, including leukemia, prostate cancer and breast cancer. miR$125 \mathrm{~b}$ has a role as an oncomiR or tumor suppressor. ${ }^{41,42}$ Shi et al. found that miR-125b promoted the growth of prostate cancer xenograft tumors by inhibiting apoptosis. ${ }^{43}$
MiR-125b has been reported to downregulate BAK1, thereby inducing androgen-independent growth of prostate cancer cells. ${ }^{44}$ We determined that BAK1 protein, but not mRNA was downregulated in OCT4-expressing cells (Figure 5a and Supplementary Figure 1). Likewise, western blot analysis revealed that BAK1 expression is inversely correlated with miRNA-125b expression in cervical cancer cells, regardless of the OCT4 level (Figure 5c). In addition, a series of luciferase report assay also demonstrated that BAK1 is one of the direct targets of miR-125b in accordance with previous studies and OCT4 inhibited BAK1 expression through miR-125b (Figure $5 \mathrm{~d}$ ). These findings suggest that OCT4 acts indirectly on BAK1 to mediate its downregulation and subsequent inhibition of apoptosis. Furthermore, IHC analyses of cervical carcinoma tissue sections revealed that BAK1 expression in primary cervical cancers is negatively correlated with the expression of OCT4 (Figures $5 \mathrm{e}$ and f). In contrast, miR-125b and OCT4 were positively correlated (Figure $5 \mathrm{~g}$ ), further suggesting that OCT4 reactivation may be involved in cervical carcinogenesis through the miRNA-125b/ BAK1 pathway.

In conclusion, the current report links the expression of the pluripotent transcription factor OCT4 to miRNA-125b in cervical carcinoma cells. Ectopic OCT4 activates miR125b-1, which, in turn, inhibits BAK1 translation. Reduced BAK1 expression inhibits apoptosis and promotes tumor progression. As modeled in Figure 6, we suggest the involvement of the OCT4/miR-125b/BAK1 pathway in human cervical carcinogenesis. To our knowledge, this is the first study on the mechanism by which OCT4 deregulation contributes to cervical cancer. These findings not only provide insight into the interplay between OCT4 and miR-125b-1 during cervical carcinogenesis, but also open up new perspectives concerning OCT4 function and regulation under normal and pathological conditions.

\section{Materials and Methods}

Cell lines and clinical samples. Three human cervical cancer cell lines (HeLa, SiHa and C-33 A) and two teratoma cell lines (Tera-1, PA-1) were from the American Type Culture Collection. The cervical cancer cell lines and PA-1 were maintained in high-glucose Dulbecco's modified Eagle's medium (Sigma-Aldrich, St. Louis, MO, USA) with 10\% fetal bovine serum (Invitrogen, Carlsbad, CA, USA). Tera-1 cells were cultured in McCoy's 5A Medium (Sigma-Aldrich) with 15\% fetal bovine serum. All cell lines were incubated at $37^{\circ} \mathrm{C}$ in a humidified $5 \% \mathrm{CO}_{2}$ atmosphere.

Clinical specimens $(n=106)$ were obtained from 42 patients with NC epithelia (NC), 20 patients with CIS and 44 patients with ICC at the Medicine School of Xi'an Jiaotong University between 2005 and 2010. None of the patients had received chemotherapy, immunotherapy or radiotherapy before specimen collection. Clinical stage and histological classifications were based on the International Federation of Gynecology and Obstetrics classification system. And this study was approved by the Internal Review Board of Medical School of Xi'an Jiaotong University.

IHC. IHC was performed on $4-\mu \mathrm{m}$ sections prepared from formalin-fixed, paraffin-embedded tissues. Briefly, tissue sections were successively deparaffinized and rehydrated, followed by treatment with citrate buffer $(2 \mathrm{mM}$ citric acid and $10 \mathrm{mM}$ sodium citrate, $\mathrm{pH} \mathrm{6.0)}$ in a steam pressure cooker. After treating with $3 \% \mathrm{H}_{2} \mathrm{O}_{2}$ and washing with phosphate-buffered saline (PBS) at room temperature, the sections were incubated at $4{ }^{\circ} \mathrm{C}$ with the following primary antibodies: anti-OCT4 (1:100, H-134, sc-9081, Santa Cruz Biotechnology, Santa Cruz, CA, USA) and anti-BAK1 (1:100, N-20, sc-1035, Santa Cruz Biotechnology). The sections were then incubated with horseradish peroxidase-conjugated secondary antibodies for $20 \mathrm{~min}$, visualized using $0.05 \% 3,3^{\prime}$-diaminobenzidine, and the 
nuclei were counterstained with hematoxylin. Negative controls were prepared using the same procedure, and PBS was used as a substitute for the primary antibody.

Sections were examined under an Olympus-CX31 microscope (Olympus, Tokyo, Japan) and scored by two separate investigators in five randomly selected representative fields at $\times 40$ magnification. OCT4 and BAK1 staining were evaluated using the IRS. The score was determined by multiplying the staining intensity (scored as 1, no staining; 2 , weak staining and 3 , strong staining) by the percentage of positive cells (scored as 1, 0-20\% positive cells; 2, 21-40\%; 3, $41-60 \%$; 4, 61-80\%; or 5, $81-100 \%$ ). OCT4 nuclear staining was divided into two groups: negative (1-4) and positive (5-15).

HPV infection analysis. Clinical sample DNA was extracted from 44 clinical cervical cancer samples with the Universal Genomic DNA Extraction Kit Ver3.0 (TaKaRa, Dalian, China) according to the manufacturer's instructions. The presence of HPV DNA in these samples was determined by PCR with universal primers $(\mathrm{GP} 5+/ \mathrm{GP} 6+)$ for HPV. The PCR method described by de Roda Husman et al. was used. ${ }^{27}$

Plasmid construction and transfection. The human OCT4 cloning vector (pGEM-T-OCT4) was kindly provided by Professor Hai-Bin Xia (Shaanxi Normal University, Xi'an, Shaanxi province, People's Republic of China). The fulllength OCT4-coding sequence was amplified and cloned into the pCAG-IRES2AcGFP1 expression vector, which was generated by replacing the CMV promoter of pIRES2-AcGFP1 (Clontech, Mountain View, CA, USA) with the CAG promoter of the vector pCAG-GFP (Addgene, Cambridge, MA, USA).

To generate plasmids that express OCT4-specific short hairpin RNA, sequences were used as follows: shOCT4-872, 5'-TGCTGTACAGAACCACACTCGGAC CAGTTTTGGCCACTGACTGACTGGTCCGAGTGGTTCTGTA-3' ${ }^{\prime}$ and $5^{\prime}$-CCTGTA CAGAACCACTCGGACCAGTCAGTCAGTGGCCAAAACTGGTCCGAGTGTGGTT CTGTAC-3'; shOCT4-911, 5'-TGCTGCATAGTCGCTGCTTGATCGCTGTTTTGG CCACTGACTGACAGCGATCACAGCGACTATG-3' and 5'-CCTGCATAGTCGCT GTGATCGCTGTCAGTCAGTGGCCAAAACAGCGATCAAGCAGCGACTATGC-3' Double oligonucleotides were cloned into pcDNA6.2 vector (Invitrogen). The pcDNA6.2-GW/EmGFP-miR-neg control plasmid was used as the negative control.

The pCAG-GFP-125b-1 expression vector was generated by cloning a fragment (extending from position -36 to +183 bp relative to the $5^{\prime}$-end of pre-miR125b-1) into the Bsp1407l/Notl sites of the pCAG-GFP-neo plasmid. Synthesized 45-nt DNA oligonucleotides containing two copies of the MRE were annealed and ligated into EcoRI/BamHI sites of the pCAG-Sponge-Ready vector established in our lab to generate the sponge plasmid pCAG-125b-2 $\times$ sponge. The plasmid pCAG-125b- $6 \times$ sponge containing six copies of binding sites was generated using the isocaudamer of Spel/Nhel.

For promoter analyses, both a fragment (from position -5000 to -1 bp relative to the $5^{\prime}$-end of pre-miR-125b-1) and a series of $5^{\prime}$ deletion mutants were cloned into the pGL3-Basic Vector (Promega, Madison, WI, USA) to generate miR-125b-1 promoter reporter constructs. A potential OCT4 RR1 was artificially subcloned into the Kpnl/Sacl sites of the pro-miR-125b-1-300 and pro-miR125b-1-700 vectors, in which two sites were purposely designed to accommodate the primers.

To construct a reporter vector containing the wild-type $3^{\prime}$-UTR of BAK1 mRNA, a $1.25-\mathrm{kb}$ fragment of the $3^{\prime}$-UTR of BAK1 mRNA extracted from HeLa cells was amplified by PCR, and cloned into the PMIR-REPORT luciferase vector downstream of the Luciferase gene. For mutation analysis, the 8-nt core seed sequences on the BAK1 $3^{\prime}$-UTR (TCTCAGGG) was replaced by random bases (GAGACAAA), whereas for deletion analysis, the fragment (from position 1-628 bp relative to the $5^{\prime}$-end of BAK1 $3^{\prime}-$ UTR), which does not contains the core seed sequences, was cloned into the pMIR-REPORT luciferase vector.

The primers and oligonucleotides are listed in Supplementary Table 1. Restriction enzymes were obtained from TaKaRa. All constructs were verified by sequencing.

To generate stable transfected cell lines, cells were transfected using Lipofectamine 2000 Reagent (Invitrogen) according to the manufacturer's instructions. Individual drug-resistant clones were isolated under treatment with $1000 \mu \mathrm{g} / \mathrm{ml}$ G418 reagent (Calbiochem, La Jolla, CA, USA) and identified by western blotting.

RNA analysis. Total RNA was extracted from cultured cells and clinical samples with TRIzol reagent (Invitrogen). For pri-miR-125b-1, pri-miR-125b-2 and other apoptosis-related genes, reverse transcription-PCR (RT-PCR) was performed using an RT-PCR kit (MBI, Hanover, MD, USA). Quantitative real-time RT-PCR was performed in an iQ5 Real-Time PCR Detection System (Bio-Rad, Hercules, CA, USA) using SYBR Premix Ex Taq II (TaKaRa). The primers used for this study are listed in Supplementary Table 1. The results were analyzed according to the CT $(\Delta \Delta \mathrm{Ct})$ method using GAPDH as the normalizing gene.

For mature miRNAs, approximately $0.05 \mathrm{mg}$ of total RNA was reversetranscribed using the Taqman MicroRNA Reverse Transcription Kit (Applied Biosystems, Foster City, CA, USA). Comparative real-time PCR was performed in triplicate using the Taqman Universal PCR Master Mix (Applied Biosystems). Relative expression levels were calculated according to the comparative CT method, using RNU6B for normalization.

Western blot analysis. Cells and clinical tissues were lysed on ice in lysis buffer (50 mM Tris-HCl, pH 7.4; 150 mM NaCl; 2 mM EDTA; 1\% NP-40; and 0.1\% SDS) containing freshly added protease inhibitor cocktail (Complete Mini; Roche Diagnostics, Branchburg, NJ, USA). Protein concentrations were measured using the BCA Protein Assay Reagent (Pierce, Rockford, IL, USA), and $30 \mu \mathrm{g}$ of total protein were separated by SDS-PAGE and blotted to activated polyvinylidene difluoride membranes (Millipore, Billerica, MA, USA). After blocking with 5\% fat-free milk, the membranes were incubated with primary antibodies as follows: anti-OCT4 (1:500, H-134, sc-9081, Santa Cruz Biotechnology), anti-BAK1 (1:500, N-20, sc-1035, Santa Cruz Biotechnology) and anti- $\beta$-actin (1:500, C4, sc-47778, Santa Cruz Biotechnology). Blots were incubated with a secondary antibody coupled to horseradish peroxidase (Thermo Fisher Scientific Inc., New York, NY, USA), followed by ECL detection (Millipore) and visualization on X-ray film. Relative quantitation was determined with the AlphaView system (Cell Biosciences, Santa Clara, CA, USA), using $\beta$-actin as the loading control.

Animal and tumor xenograft assay. To assess in vivo tumorigenicity, exponentially growing cells were bilaterally inoculated into subcutaneous sites of 4- to 6-week-old Balb/c athymic nude mice. Approximately $200 \mu \mathrm{l}$ of medium containing $1 \times 10^{6}$ cells and resuspended in 50\% Matrigel (BD Biosciences, San Jose, CA, USA) were used for inoculating. Tumor dimensions were measured with calipers once every 4 days, and volumes $\left(\mathrm{cm}^{3}\right)$ were calculated according to the standard formula: length $\times$ width $^{2} / 2$. At the end of the experiment, tumors were dissected out, and the net weight per mouse was measured. The experimental protocols used herein were evaluated and approved by the Animal Care and Use Committee of the Medical School of Xi'an Jiaotong University.

Apoptosis and flow cytometry analysis. Apoptosis was analyzed in vitro using the FACS Annexin V assay kit (BD Biosciences) according to the manufacturer's instructions. Briefly, cells were harvested at $1 \times 10^{5}$ cells per tube, and stained in duplicate with $5 \mu$ of APC-Annexin V conjugate and $5 \mu$ l of propidium iodide $(10 \mu \mathrm{g} / \mathrm{ml})$ for $30 \mathrm{~min}$ in the dark. Subsequently, the samples were analyzed by a FACS Calibur flow cytometry (Becton Dickinson, Franklin Lakes, NJ, USA). The percent of apoptotic cells is referred to as the apoptotic index.

TUNEL assay. Paraffin-embedded tissue slides were prepared from the xenograft tumors. TUNEL staining was detected by the DEAD END Colorimetric TUNEL System (Promega) according to the manufacturer's instruction. Apoptotic nuclei were analyzed by counting the total number of TUNEL-positive nuclei, excluding cells undergoing mitosis in 10 random fields.

Luciferase reporter assay. Briefly, plasmids containing firefly luciferase reporters were cotransfected into cells using Lipofectamine 2000 (Invitrogen). After $48 \mathrm{~h}$, the cell monolayers were harvested and resuspended in PLB buffer. Luciferase activity was measured with a luminometer (Promega), and the transfection efficiency was normalized to the paired Renilla luciferase activity using the Dual Luciferase Reporter Assay system (Promega). Specific activity is shown as the fold change of the experimental group versus the control group.

Quantitative ChIP assay. HeLa and SiHa cells were subjected to ChIP with the EZ-ChIP Assay kit (Millipore). Briefly, cells were treated with $37 \%$ formaldehyde to crosslink proteins, and terminated with $0.125 \mathrm{M}$ glycine. After being performed with sonication, chromatin-protein complexes were immunoprecipitated with $5 \mu \mathrm{g}$ of anti-OCT4 antibodies (sc-9081, Santa Cruz Biotechnnology) or $1 \mu \mathrm{g}$ of mouse $\mathrm{lgG}$. Real-time PCR was performed to amplify the regions of interest or internal negative control regions. The primers used for these studies are listed in Supplementary Table 1. The fold enrichment ratio was calculated as the value of the ChIP sample versus the corresponding input sample. Samples yielding a twofold enrichment or better were considered positive targets. 
Statistical analysis. Statistical analyses were performed with the Statistical Package of Social Science (SPSS) software, version 16.0 (SPSS Inc., Chicago, IL, USA). Univariate analyses were done by the Student's $t$-test or one-way ANOVA test. Difference between groups of the volume formed by xenografts was determined by two-way ANOVA test. Correlations between OCT4 and BAK1 relative expression were evaluated using the Pearson's correlation test. In all tests, a value of $P<0.05$ was considered statistically significant.

\section{Conflict of Interest}

The authors declare no conflict of interest.

Acknowledgements. We thank Professor Hai-Bin Xia (Shaanxi Normal University, Xi'an, Shaanxi province, People's Republic of China) for the generous gift of pGEM-T-OCT4 plasmid. This work was supported by the National Natural Science Foundation of China (General Program, No. 30571951), the National Natural Science Fund for Distinguished Young Scientists (No. 30725043) and the 985-2 Project of the Ministry Education (cancer stem cell).

1. Ma YY, Wei SJ, Lin YC, Lung JC, Chang TC, Whang-Peng J et al. PIK3CA as an oncogene in cervical cancer. Oncogene 2000; 19: 2739-2744.

2. Paavonen J. Human papillomavirus infection and the development of cervical cancer and related genital neoplasias. Int J Infect Dis 2007; 11(Suppl 2): S3-S9.

3. Ji J, Zheng PS. Expression of Sox2 in human cervical carcinogenesis. Hum Pathol 2010; 41: 1438-1447.

4. Wen J, Park JY, Park KH, Chung HW, Bang S, Park SW et al. Oct4 and Nanog expression is associated with early stages of pancreatic carcinogenesis. Pancreas 2010; 39: 622-626.

5. Wei D, Kanai M, Jia Z, Le X, Xie K. Kruppel-like factor 4 induces p27Kip1 expression in and suppresses the growth and metastasis of human pancreatic cancer cells. Cancer Res 2008; 68: 4631-4639.

6. Scholer HR, Ruppert S, Suzuki N, Chowdhury K, Gruss P. New type of POU domain in germ line-specific protein Oct-4. Nature 1990; 344: 435-439.

7. Hansis C, Grifo JA, Krey LC. Oct-4 expression in inner cell mass and trophectoderm of human blastocysts. Mol Hum Reprod 2000; 6: 999-1004.

8. Loh YH, Wu Q, Chew JL, Vega VB, Zhang WW, Chen X et al. The Oct4 and Nanog transcription network regulates pluripotency in mouse embryonic stem cells. Nat Genet 2006; 38: 431-440.

9. Palmieri SL, Peter W, Hess H, Scholer HR. Oct4 transcription factor is differentially expressed in the mouse embryo during establishment of the first two extraembryonic cell lineages involved in implantation. Dev Biol 1994; 166: 259-267.

10. Niwa H, Miyazaki J, Smith AG. Quantitative expression of Oct-3/4 defines differentiation, dedifferentiation or self-renewal of ES cells. Nat Genet 2000; $24: 372-376$.

11. Kuroda T, Tada M, Kubota H, Kimura H, Hatano SY, Suemori $\mathrm{H}$ et al. Octamer and Sox elements are required for transcriptional cis regulation of Nanog gene expression. Mol Cell Biol 2005; 25: 2475-2485.

12. Nishimoto M, Fukushima A, Okuda A, Muramatsu M. The gene for the embryonic stem cell coactivator UTF1 carries a regulatory element which selectively interacts with a complex composed of Oct-3/4 and Sox-2. Mol Cell Biol 1999; 19: 5453-5465.

13. Tokuzawa Y, Kaiho E, Maruyama M, Takahashi K, Mitsui K, Maeda M et al. Fbx15 is a novel target of Oct3/4 but is dispensable for embryonic stem cell self-renewal and mouse development. Mol Cell Biol 2003; 23: 2699-2708.

14. Tomioka M, Nishimoto M, Miyagi S, Katayanagi T, Fukui N, Niwa $\mathrm{H}$ et al. Identification of Sox-2 regulatory region which is under the control of Oct-3/4-Sox-2 complex. Nucleic Acids Res 2002; 30: 3202-3213.

15. Card DA, Hebbar PB, Li L, Trotter KW, Komatsu Y, Mishina Y et al. Oct4/Sox2-regulated miR-302 targets cyclin D1 in human embryonic stem cells. Mol Cell Biol 2008; 28: 6426-6438.

16. Takahashi K, Tanabe K, Ohnuki M, Narita M, Ichisaka T, Tomoda K et al. Induction of pluripotent stem cells from adult human fibroblasts by defined factors. Cell 2007; 131: 861-872.

17. Hochedlinger K, Yamada Y, Beard C, Jaenisch R. Ectopic expression of Oct-4 blocks progenitor-cell differentiation and causes dysplasia in epithelial tissues. Cell 2005; 121 : $465-477$.

18. Cheng L. Establishing a germ cell origin for metastatic tumors using OCT4 immunohistochemistry. Cancer 2004; 101: 2006-2010.

19. Jones TD, Ulbright TM, Eble JN, Baldridge LA, Cheng L. OCT4 staining in testicular tumors: a sensitive and specific marker for seminoma and embryonal carcinoma. Am J Surg Pathol 2004; 28: 935-940.
20. Looijenga LH, Stoop H, de Leeuw HP, de Gouveia Brazao CA, Gillis AJ, van Roozendaal KE et al. POU5F1 (ОСТ3/4) identifies cells with pluripotent potential in human germ cell tumors. Cancer Res 2003; 63: 2244-2250.

21. Amin R, Mishra L. Liver stem cells and tgf-Beta in hepatic carcinogenesis. Gastrointest Cancer Res 2008; 2: S27-S30.

22. Ezeh UI, Turek PJ, Reijo RA, Clark AT. Human embryonic stem cell genes OCT4, NANOG, STELLAR, and GDF3 are expressed in both seminoma and breast carcinoma. Cancer Am Cancer Soc 2005; 104: 2255-2265.

23. Liu CG, Lu Y, Wang BB, Zhang YJ, Zhang RS, Lu Y et al. Clinical implications of stem cell gene Oct-4 expression in breast cancer. Ann Surg 2011; 253: 1165-1171.

24. Atlasi Y, Mowla SJ, Ziaee SA, Bahrami AR. OCT-4, an embryonic stem cell marker, is highly expressed in bladder cancer. Int J Cancer 2007; 120: 1598-1602.

25. Chang CC, Shieh GS, Wu P, Lin CC, Shiau AL, Wu CL. Oct-3/4 expression reflects tumor progression and regulates motility of bladder cancer cells. Cancer Res 2008; 68: 6281-6291.

26. Chang JH, Au HK, Lee WC, Chi CC, Ling TY, Wang LM et al. Expression of the pluripotent transcription factor OCT4 promotes cell migration in endometriosis. Fertil Steril 2013; 99: 1332-1339, e1335.

27. de Roda Husman AM, Walboomers JM, van den Brule AJ, Meijer CJ, Snijders PJ. The use of general primers GP5 and GP6 elongated at their 3' ends with adjacent highly conserved sequences improves human papillomavirus detection by PCR. J Gen Virol 1995; 76(Pt 4): 1057-1062.

28. Chang CJ, Chien Y, Lu KH, Chang SC, Chou YC, Huang CS et al. Oct4-related cytokine effects regulate tumorigenic properties of colorectal cancer cells. Biochem Bioph Res Co 2011; 415: 245-251.

29. Hu TS, Liu SR, Breiter DR, Wang F, Tang Y, Sun SH. Octamer 4 small interfering RNA results in cancer stem cell-like cell apoptosis. Cancer Res 2008; 68: 6533-6540.

30. Guo Y, Mantel C. Hromas RA, Broxmeyer HE. Oct-4 is critical for survival/antiapoptosis of murine embryonic stem cells subjected to stress: Effects associated with Stat3/Survivin. Stem Cells 2008; 26: 30-34.

31. Bousquet M, Quelen C, Rosati R, Mansat-De Mas V, La Starza R, Bastard C et al. Myeloid cell differentiation arrest by miR-125b-1 in myelodysplastic syndrome and acute myeloid leukemia with the $\mathrm{t}(2 ; 11)(\mathrm{p} 21 ; \mathrm{q} 23)$ translocation. J Exp Med 2008; 205: 2499-2506.

32. Klusmann JH, Li Z, Bohmer K, Maroz A, Koch ML, Emmrich $S$ et al. miR-125b-2 is a potential oncomiR on human chromosome 21 in megakaryoblastic leukemia. Genes Dev 2010; 24: 478-490.

33. Zhou M, Liu Z, Zhao Y, Ding Y, Liu H, Xi Y et al. MicroRNA-125b confers the resistance of breast cancer cells to paclitaxel through suppression of pro-apoptotic $\mathrm{Bcl}-2$ antagonist killer 1 (Bak1) expression. J Biol Chem 2010; 285: 21496-21507.

34. Yuan FJ, Zhou WB, Zou C, Zhang ZY, Hu HS, Dai ZQ et al. Expression of Oct4 in HCC and modulation to wnt/beta-catenin and TGF-beta signal pathways. Mol Cell Biochem 2010; 343: 155-162.

35. Chen Z, Xu WR, Qian H, Zhu W, Bu XF, Wang S et al. Oct4, a Novel Marker for Human Gastric Cancer. J Surg Oncol 2009; 99: 414-419.

36. Ovitt CE, Scholer HR. The molecular biology of Oct-4 in the early mouse embryo. Mol Hum Reprod 1998; 4: 1021-1031.

37. Cheng L, Thomas A, Roth LM, Zheng W, Michael H, Karim FW. OCT4: a novel biomarker for dysgerminoma of the ovary. Am J Surg Pathol 2004; 28: 1341-1346.

38. Cantz T, Key G, Bleidissel M, Gentile L, Han DW, Brenne A et al. Absence of OCT4 expression in somatic tumor cell lines. Stem Cells 2008; 26: 692-697.

39. Kim RJ, Nam JS. OCT4 expression enhances features of cancer stem cells in a mouse model of breast cancer. Lab Anim Res 2011; 27: 147-152.

40. Chen TJ, Du J, Lu GX. Cell growth arrest and apoptosis induced by Oct4 or Nanog knockdown in mouse embryonic stem cells: a possible role of Trp53. Mol Biol Rep 2012; 39: $1855-1861$.

41. Bousquet M, Harris MH, Zhou B, Lodish HF. MicroRNA miR-125b causes leukemia. Proc Natl Acad Sci USA 2010; 107: 21558-21563.

42. Guan $Y$, Yao H, Zheng Z, Qiu G, Sun K. MiR-125b targets BCL3 and suppresses ovarian cancer proliferation. Int $J$ Cancer 2011; 128: 2274-2283.

43. Shi XB, Xue L, Ma AH, Tepper CG, Kung HJ, White RW. miR-125b promotes growth of prostate cancer xenograft tumor through targeting pro-apoptotic genes. Prostate 2011; 71: $538-549$

44. Shi XB, Xue L, Yang J, Ma AH, Zhao J, Xu M et al. An androgen-regulated miRNA suppresses Bak1 expression and induces androgen-independent growth of prostate cancer cells. Proc Natl Acad Sci USA 2007; 104: 19983-19988.

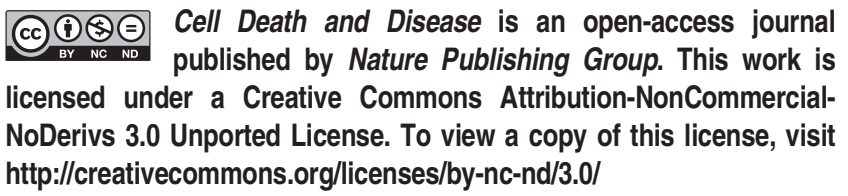

